\title{
Independent Variables Affecting Employee Behaviour in Socially Responsible Organisations: Working Environment in Lithuania and Poland
}

\author{
Jolita Vveinhardt ${ }^{1}$, Wlodzimierz Sroka $\mathbf{2}^{2,3^{*}}$ \\ ${ }^{1}$ Vytautas Magnus University \\ K. Donelaicio st. 58, LT-44248, Kaunas, Lithuania \\ E-mail.jolita.vveinhardt@vdu.lt \\ ${ }^{2}$ WSB University \\ Cieplaka 1c, 41-300 Dabrowa Gornicza, Poland \\ ${ }^{3}$ University of Johannesburg, College of Business and Economics \\ JBS Towers, Cnr Barry Hertzog and Empire Roads, Milpark, Johannesburg, South Africa \\ E-mail.wsroka@wsb.edu.pl \\ * corresponding author
}

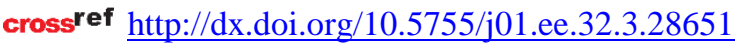

\begin{abstract}
Although the improvement of work environment is often associated with greater operational efficiency, we propose looking at it from a different angle. The improvement of working conditions creates a "side effect" that provides significant support for the implementation of CSR goals. That is, organizations have the opportunity not only to improve accountability to this stakeholder group, but also to stimulate its socially responsible behaviour. The purpose of this paper is to identify independent variables affecting employee behaviour in socially responsible organisations. To conduct the research, a questionnaire survey method was chosen. The research sample includes 823 respondents from Lithuania and Poland. The research hypotheses were tested by performing the linear regression analysis with independent variables. Our findings show that when such features as employee intentions to leave the job, the extremity of work content, biased work management, environment that does not correspond to ergonomic requirements, and poor working conditions were decreasing while other variables in Lithuanian organisations remained unchanged, the socially responsible behaviour of the organisations was strengthening. In Poland's case, as negative intentions and attitudes of employees were weakening, the nature of tasks and working conditions were becoming more acceptable, while other social variables remained unchanged, the employee's socially responsible behaviour was also strengthening.
\end{abstract}

Keywords: Corporate Social Responsibility; Organisation's Behaviour; Employee's Behaviour; Working Environment; Lithuania; Poland.

\section{Introduction}

A good working environment supports safety of employees, company's growth, and target achievement. It is maintained that such an environment is the best to successful workforce because it encourages employees to perform to their highest ability (Ahmad et al., 2019; Nahrgang et al., 2011, Wang et al., 2021). If this is so, how can companies achieve it? Some claim that companies can do it by focusing on their overall culture, supporting employee growth, and making employees feel safe and comfortable (Khan et al., 2018; Ostroff et al., 2012); therefore, organizations should eliminate negative factors such as ostracism and harassment in order to increase work efficiency to ensure their success (Anjum et al., 2018). We are of the opinion that one of the key aspects is social responsibility and being a socially responsible organisation. Unfortunately, there are many organisations that treat the concept of CSR as a matter of fashion and benefit. This is a negative factor as CSR positively affects job satisfaction, organisational commitment, and organizational loyalty (Lee \& Park, 2013), and without CSR activities, business may lose potential staff, value to employees, and even attractiveness to the society. However, in many such organisations the employee remains a 'tool' to achieve the target. We state that the organisation's socially responsible behaviour is impossible if the employees themselves do not behave in socially responsible ways. The employees' sense of responsibility and willingness to be socially responsible depend on how they are treated and what working conditions they have in their workplace. CSR can also help to reduce turnover of employees in organisations (Bhattacharya, 2017). Given these facts, the research question of our study is: Which independent variables have the greatest impact on the behaviour of employees of socially responsible organisations, and the improvement/weakening of which factors strengthen corporate social responsibility? The purpose of the study is to identify independent variables affecting employee behaviour in socially responsible organisations. In this case, the following factors were distinguished as independent variables: employee interrelationships including communication, isolation, reputation, demographic peculiarities, their views, experienced harm, emotional state, and intentions; nature of tasks, work content; work organisation; and physical working environment.

Our study is organised as follows. Its first part presents the theoretical background of CSR and employee behaviour, 
which constitutes the base for the formulation of research hypotheses. In turn, section two presents materials and methods applied in our survey. This is followed by findings and their analysis. Finally, conclusions, directions for further research and limitations of our paper are presented. In this article, authors present only a part of the results of the conducted study.

\section{Theoretical Background}

CSR has become a leading corporate strategy worldwide after discovering CSR principles as a major strategic force (Bhattacharya, 2017) gradually advancing from philanthropic programs to authentic strategies intended to regain trust of the society and creating value for shareholders (Aluchna \& Roszkowska-Menkes, 2019). In general, it means that firms should meet the expectations of their interest groups (Goel \& Ramanathan, 2014), behave in accordance with the CSR concept (Sroka \& Lorinczy, 2015; Czubala, 2016), and create a positive impact on the society, environment, and economy. The core value of CSR is then to maintain the economic aspects of the organization while balancing the environmental and social issues (Sroka \& Vveinhardt, 2020 a). In other words, the organisation should behave responsibly (Mohr et al., 2001). Relevance of the concept of CSR is confirmed by problems identified in the business sphere, such as reprehensible behaviour towards customers and employees, lack of responsibility for the impact on the environment, and the negative effects of inadequate organisational management (Majerova, 2015). However, it should be added that CSR also applies to public sector organisations because CSR helps to identify and codify organisational values, determine the organisation's identity, and structure its decisions (Toleikiene \& Jukneviciene, 2019), as well as to SMEs companies: CSR tends to be less formal and carried out intuitively, without any prescribed management systems or models (Salciuviene et al., 2016). Despite these facts, the level of awareness of the CSR concept in CEE still remains 'far from excellent' when compared to Western countries, though companies' CSR engagement should be regarded as one of the central parts of communication with their stakeholders (Vveinhardt et al., 2019), even though CSR investment doesn't always guarantee positive financial outcomes (Kyun Chang et al., 2021).

According to Sheehy (2015), despite lengthy debates, business and academic communities and other stakeholders have thus far failed to reach consensus on what constitutes the content of CSR. The political, legal, social and economic peculiarities of states contribute to the lack of agreement (Chapardar \& Khanlari, 2011). Research conducted in recent decades shows that, in general, enterprises are moving away from the existing understanding of values of social responsibility, turning it into formal practice that does not create long-term value for stakeholders (Rajak, 2008; Cheshire, 2010), which leads to inducements to strengthen the moral dimension of CSR (Moratis, 2016; 2018). This is especially important given the fact that people spend a substantial part of their life at work. As Khan et al. (2020) state, CSR is a "key human life, social and economic development". CSR values are of particular concern to employees, to whom safe working conditions must be created. Social responsibility is defined as encompassing all aspects of the design and management of the work system that affect employees' interactions with the workplace (Searcy et al., 2016). As Neumann et al. (2014) claim, this can include "the physical design, including layouts and the built environment, division of labour, use of technology, supervisory structures, human resource management strategies, and co-worker interactions that can affect an employee's physical, mental, and emotional workload and determine the positive or negative outcomes of work for the employee". Working conditions play an important role, as they can affect work performance and psychological wellbeing of employees, and socially responsible actions can improve the identification of employees with the organisation (Gkorezis \& Petridou, 2017). Because conflicts are practically inevitable in every organisation, everything must be done to mitigate their negative effects.

Normative documents of Western countries, describing working conditions, pay considerable attention to working conditions regulating employees' judicial guarantees and physical and psychological comfort; therefore, it is important that enterprises also understand the ethical aspect of the welfare of employees as stakeholders (O'Connor and Shumate, 2010). Employee welfare can be ensured by a healthy organisational climate characterised not only by the physical parameters of the safe environment created by the organisation but also by the psychological state, quality of internal communication, perception and experience of definiteness, and leadership ethics (De Roeck \& Farooq, 2018; Hansen et al., 2016; Kuenzi \& Schminke, 2009; Wang \& Hsieh, 2013; Verdorfer et al., 2013). All of it gives meaning to the organisation's responsibility to meet the expectations of employees as stakeholders and strengthens their own propensity to greater social good (De Roeck \& Farooq, 2018). On the other hand, shortcomings of participation in decision-making and of skills in resolving disagreements, inadequate safety, and emerging ambiguities regarding work tasks create a negative working environment.

Looking through the prism of CSR enables coping with the problems arising in the management of the organisation too. It should be added that, although there are opponents of CSR (see Chwistecka-Dudek, 2016), social responsibility also improves the climate in the organisation (Garavan et al., 2010) as well as causes some other positive effects; e.g., it reduces chances for mobbing occurrence (Lee, 2020). The probability that mobbing can be avoided is higher if organisations are trustworthy and behave ethically (Khan et al., 2020). In turn, harmful organisational culture generates favourable conditions for occurrence of mobbing (LutgenSandvik \& Tracy, 2012).

Research also shows that the development of CSR commitments to internal stakeholders is an important condition for employees' physical and psychological wellbeing (Macassa et al., 2020; O’Connor \& Shumate, 2010; Androniceanu, 2019). Other authors accentuate the ethical aspects of work organisation and interpersonal relationships (Akar, 2018; Leymann, 1990; McCarthy et al., 2003; Ranki et al., 2018). Although the concept of CSR provides for high standards of moral conduct, Rose (2007) concluded that additional ethics training had not significantly influenced the decisions of leaders of many organisations. That is not good, given that ethical leadership can stimulate employees' CSR engagement through creating a better employee-organisation 
fit (Nejati et al., 2019). Therefore, formalised ethical norms are needed (Petrescu \& Manghiuc, 2020). Nevertheless, McCarthy et al. (2003), who analysed studies on CSR, underline the lack of organisational awareness of incurred costs and benefits that would be obtained from more socially responsible actions. This problem is also highlighted by Androniceanu and Tvaronaviciene (2019).

Another issue raised by the researchers is the nature of activities undertaken by organisations. In addition, the nature of those activities determine the tasks imposed on employees, especially if they are related to change. It should be underlined, however, that that change itself is not at fault but inadequate management responses to change, going beyond the limits of norms, are blameworthy.

Given the deliberations presented, the following hypotheses were formed:

H1. Along with the improvement of factors related to employee relationships, the socially responsible organisation's behaviour is strengthening (improving).

H2. Along with the improvement of factors related to work management, the socially responsible organisation's behaviour is strengthening (improving).

H3. Along with the improvement of factors related to the working environment and working conditions, the socially responsible organisation's behaviour is strengthening (improving).

H4. Along with the improvement of factors related to the nature of tasks, the socially responsible behaviour of employees is strengthening (improving).

\section{Materials and Methods}

\section{Procedure and Sample}

The research sample was formed by choosing two geographically, historically and culturally close countries Lithuania and Poland. Prior to starting the research, organisations were selected and managers and/or other responsible persons were addressed regarding the permission to conduct the survey in the organisations they led. The survey was conducted in 2019. The invitations to participate in the survey contained detailed description of the idea of the research and its purpose, and data protection was guaranteed. The researchers comprehensively protected the research participants' anonymity and guaranteed confidentiality. The study involved 823 employees from organisations of both countries. The questionnaire survey was conducted remotely; i.e., by distributing an electronic survey link.

\section{Measures}

To conduct the survey, the questionnaire survey method was chosen. The psychometric characteristics of the questionnaire are detailed in another article written by the authors (see: Vveinhardt \& Sroka, 2020a, 2020b). We must note that the questionnaire used for the survey is valid and reliable. Correlation of the relationships between the subscales of the questionnaire, presented in Table 1, shows interconnectedness and statistically reliable relationships, except for work content vs. environmental responsibility. The factor structure of this research constructs was examined by means of exploratory factor analysis (EFA) (Table 2).

Intercorrelations among Subscales

\begin{tabular}{|c|c|c|c|c|c|c|c|c|c|}
\hline \multirow{3}{*}{\multicolumn{2}{|c|}{ Scales and subscales }} & \multirow{3}{*}{ 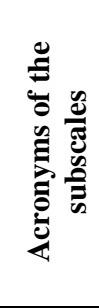 } & \multicolumn{7}{|c|}{ Corporate Social Responsibility } \\
\hline & & & \multicolumn{5}{|c|}{ FOSB } & \multicolumn{2}{|c|}{ FESB } \\
\hline & & & 预 & 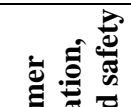 & 焉 & 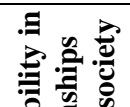 & 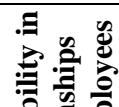 & \& & \& \\
\hline \multirow{16}{*}{ FEIR } & \multirow{2}{*}{$\begin{array}{l}\text { Employee } \\
\text { communication }\end{array}$} & \multirow{2}{*}{ ECO } & $-0.412 * *$ & $-0.408 * *$ & $-0.233 * *$ & $-0.336 * *$ & $-0.408 * *$ & $-0.370 * *$ & $-0.154 * *$ \\
\hline & & & 0.000 & 0.000 & 0.000 & 0.000 & 0.000 & 0.000 & 0.000 \\
\hline & \multirow{2}{*}{ Employee isolation } & \multirow{2}{*}{ EIS } & $-0.455 * *$ & $-0.462 * *$ & $-0.274 * *$ & $-0.372 * *$ & $-0.458 * *$ & $-0.403 * *$ & $-0.169 * *$ \\
\hline & & & 0.000 & 0.000 & 0.000 & 0.000 & 0.000 & 0.000 & 0.000 \\
\hline & \multirow{2}{*}{ Employee reputation } & \multirow{2}{*}{ ERE } & $-0.431 * *$ & $-0.443 * *$ & $-0.298 * *$ & $-0.378 * *$ & $-0.479 * *$ & $-0.384 * *$ & $-0.142 * *$ \\
\hline & & & 0.000 & 0.000 & 0.000 & 0.000 & 0.000 & 0.000 & 0.000 \\
\hline & \multirow{2}{*}{ Employee demography } & \multirow{2}{*}{ EDE } & $-0.402 * *$ & $-0.402 * *$ & $-0.227 * *$ & $-0.315 * *$ & $-0.359 * *$ & $-0.431 * *$ & $-0.182 * *$ \\
\hline & & & 0.000 & 0.000 & 0.000 & 0.000 & 0.000 & 0.000 & 0.000 \\
\hline & \multirow{2}{*}{ Employee views } & \multirow{2}{*}{ EVS } & $-0.361 * *$ & $-0.367 * *$ & $-0.223 * *$ & $-0.311 * *$ & $-0.330 * *$ & $-0.404 * *$ & $-0.134 * *$ \\
\hline & & & 0.000 & 0.000 & 0.000 & 0.000 & 0.000 & 0.000 & 0.000 \\
\hline & \multirow{2}{*}{$\begin{array}{l}\text { Damage experienced by } \\
\text { employees }\end{array}$} & \multirow{2}{*}{ EED } & $-0.449 * *$ & $-0.493 * *$ & $-0.237 * *$ & $-0.320 * *$ & $-0.429 * *$ & $-0.449 * *$ & $-0.192 * *$ \\
\hline & & & 0.000 & 0.000 & 0.000 & 0.000 & 0.000 & 0.000 & 0.000 \\
\hline & \multirow{2}{*}{$\begin{array}{l}\text { Employees' emotional } \\
\text { state }\end{array}$} & \multirow{2}{*}{ EES } & $-0.455 * *$ & $-0.506 * *$ & $-0.348 * *$ & $-0.387 * *$ & $-0.529 * *$ & $-0.387 * *$ & $-0.159 * *$ \\
\hline & & & 0.000 & 0.000 & 0.000 & 0.000 & 0.000 & 0.000 & 0.000 \\
\hline & \multirow{2}{*}{ Employee intentions } & \multirow{2}{*}{ EIN } & $-0.509 * *$ & $-0.555 * *$ & $-0.408 * *$ & $-0.442 * *$ & $-0.590 * *$ & $-0.405 * *$ & $-0.187 * *$ \\
\hline & & & 0.000 & 0.000 & 0.000 & 0.000 & 0.000 & 0.000 & 0.000 \\
\hline \multirow{6}{*}{ FNCA } & \multirow{2}{*}{ Nature of tasks } & \multirow{2}{*}{ WTA } & $-0.365 * *$ & $-0.383 * *$ & $-0.146^{* *}$ & $-0.290 * *$ & $-0.342 * *$ & $-0.418 * *$ & $-0.264 * *$ \\
\hline & & & 0.000 & 0.000 & 0.000 & 0.000 & 0.000 & 0.000 & 0.000 \\
\hline & \multirow{2}{*}{ Work content } & \multirow{2}{*}{ WCT } & $-0.135 * *$ & $-0.164 * *$ & 0 & $-0.078 *$ & $-0.146 * *$ & $-0.231 * *$ & $-0.273 * *$ \\
\hline & & & 0.000 & 0.000 & 0.074 & 0.025 & 0.000 & 0.000 & 0.000 \\
\hline & \multirow{2}{*}{ Work assessment } & \multirow{2}{*}{ WAS } & $-0.430 * *$ & $-0.448 * *$ & $-0.224 * *$ & $-0.378 * *$ & $-0.452 * *$ & $-0.443 * *$ & $-0.213 * *$ \\
\hline & & & 0.000 & 0.000 & 0.000 & 0.000 & 0.000 & 0.000 & 0.000 \\
\hline
\end{tabular}


Jolita Vveinhardt, Wlodzimierz Sroka. Independent Variables Affecting Employee Behaviour in Socially Responsible...

\begin{tabular}{|c|c|c|c|c|c|c|c|c|c|}
\hline \multirow{3}{*}{\multicolumn{2}{|c|}{ Scales and subscales }} & \multirow{3}{*}{ 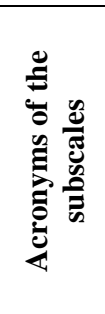 } & \multicolumn{7}{|c|}{ Corporate Social Responsibility } \\
\hline & & & \multicolumn{5}{|c|}{ FOSB } & \multicolumn{2}{|c|}{ FESB } \\
\hline & & & 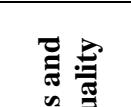 & 鳥 & 䊕 & 资 & 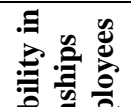 & 递电 & ¿ \\
\hline \multirow{4}{*}{ FWOM } & \multirow[b]{2}{*}{ Work organisation } & \multirow{2}{*}{ WOR } & $-0.608 * *$ & $-0.625 * *$ & $-0.457 * *$ & $-0.479 * *$ & $-0.640 * *$ & $-0.300 * *$ & $-0.073 *$ \\
\hline & & & 0.000 & 0.000 & 0.000 & 0.000 & 0.000 & 0.000 & 0.036 \\
\hline & \multirow{2}{*}{ Work management } & \multirow{2}{*}{ WMA } & $-0.626^{* *}$ & $-0.622 * *$ & $-0.491 * *$ & $-0.552 * *$ & $-0.694 * *$ & $-0.325^{* *}$ & $-0.085^{*}$ \\
\hline & & & 0.000 & 0.000 & 0.000 & 0.000 & 0.000 & 0.000 & 0.015 \\
\hline \multirow{4}{*}{ FPEC } & \multirow{2}{*}{ Working environment } & \multirow{2}{*}{ WEN } & $-0.588 * *$ & $-0.589 * *$ & $-0.523^{* *}$ & $-0.534 * *$ & $-0.621 * *$ & $-0.341 * *$ & $-0.124 * *$ \\
\hline & & & 0.000 & 0.000 & 0.000 & 0.000 & 0.000 & 0.000 & 0.000 \\
\hline & \multirow{2}{*}{ Working conditions } & \multirow{2}{*}{ WCN } & $-0.616^{* *}$ & $-0.623 * *$ & $-0.565 * *$ & $-0.585^{* *}$ & $-0.673 * *$ & $-0.301 * *$ & $-0.084 *$ \\
\hline & & & 0.000 & 0.000 & 0.000 & 0.000 & 0.000 & 0.000 & 0.016 \\
\hline
\end{tabular}

Note: Spearman's correlation coefficient: $0.6<\mathrm{r}<=0.8$ (strong relations), $0.4<\mathrm{r}<=0.6$ (moderate strength relations), $0.2<\mathrm{r}<=0.4$ (weak relations), $0.1<=\mathrm{r}<=0.2$ (very weak relations).

Acronyms of the scales: FEIR - factors related to employee interrelationship; FNCA - factors related to the nature of tasks, work content and assessment; FWOM - factors related to work organisation and management; FPEC - factors related to physical working environment and conditions; FOSB - factors related to behaviour of socially responsible organization; FESB - factors related to behaviour of socially responsible employee.

Table 2

Factor Loadings and Average Variance Extracted

\begin{tabular}{|c|c|c|c|}
\hline Abbreviations & Construct & Factor loadings & $\begin{array}{l}\text { Average } \\
\text { variance } \\
\text { extracted }\end{array}$ \\
\hline ECO & $\begin{array}{l}\text { Employee } \\
\text { communication }\end{array}$ & $\begin{array}{l}\text { ECO1 (0.810), ECO2 (0.823), ECO3 (0.867), ECO4 (0.823), ECO5 (0.849), } \\
\text { ECO6 (0.775), ECO7 (0.807). }\end{array}$ & $\mathrm{ECO}=0.822$ \\
\hline EIS & $\begin{array}{l}\text { Employee } \\
\text { isolation }\end{array}$ & $\begin{array}{l}\text { EIS1 (0.900), EIS2 (0.908), EIS3 (0.877), EIS4 (0.838), EIS5 (0.885), EIS6 } \\
(0.892) .\end{array}$ & $\mathrm{EIS}=0.883$ \\
\hline ERE & $\begin{array}{l}\text { Employee } \\
\text { reputation }\end{array}$ & $\begin{array}{l}\text { ERE1 (0.750), ERE2 (0.865), ERE3 (0.893), ERE4 (0.863), ERE5 (0.842), } \\
\text { ERE6 (0.824). }\end{array}$ & $\mathrm{ERE}=0.839$ \\
\hline EDE & $\begin{array}{l}\text { Employee } \\
\text { demography }\end{array}$ & $\begin{array}{l}\text { EDE1 (0.827), EDE2 (0.791), EDE3 (0.850), EDE4 (0.880), EDE5 (0.824), } \\
\text { EDE6 (0.832), EDE7 (0.805). }\end{array}$ & $\mathrm{EDE}=0.830$ \\
\hline EVS & Employee views & EVS1 (0.882), EVS2 (0.911), EVS3 (0.887). & $\mathrm{EVS}=0.893$ \\
\hline EED & $\begin{array}{l}\text { Damage } \\
\text { experienced by } \\
\text { employees }\end{array}$ & EED1 (0.918), EED2 (0.917), EED3 (0.888), EED4 (0.857), EED5 (0.856). & $\mathrm{EED}=0.887$ \\
\hline EES & $\begin{array}{l}\text { Employees' } \\
\text { emotional state }\end{array}$ & $\begin{array}{l}\text { EES1 (0.837), EES2 (0.825), EES3 (0.833), EES4 (0.815), EES5 (0.859), } \\
\text { EES6 (0.864), EES7 (0.825), EES8 (0.816), EES9 (0.753), EES10 (0.729), } \\
\text { EES11 (0.758), EES12 (0.739), EES13 (0.800), EES14 (0.849). }\end{array}$ & $\mathrm{EES}=0.807$ \\
\hline EIN & $\begin{array}{l}\text { Employee } \\
\text { intentions }\end{array}$ & EIN1 (0.876), EIN2 (0.933), EIN3 (0.936), EIN4 (0.881), EIN5 (0.916). & $\mathrm{EIN}=0.908$ \\
\hline WTA & Nature of tasks & $\begin{array}{l}\text { WTA1 (0.566), WTA2 (0.821), WTA3 (0.878), WTA4 (0.862), WTA5 } \\
(0.820) \text {, WTA6 (0.799), WTA7 }(0.793) .\end{array}$ & $\mathrm{WTA}=0.791$ \\
\hline WCT & Work content & WCT1 (0.838), WCT2 (0.917), WCT3 (0.910), WCT4 (0.831). & $\mathrm{WCT}=0.874$ \\
\hline WAS & $\begin{array}{l}\text { Work } \\
\text { assessment }\end{array}$ & $\begin{array}{l}\text { WAS1 (0.880), WAS2 (0.908), WAS3 (0.923), WAS4 (0.910), WAS5 } \\
(0.899) .\end{array}$ & $\mathrm{WAS}=0.904$ \\
\hline WOR & $\begin{array}{l}\text { Work } \\
\text { organisation }\end{array}$ & $\begin{array}{l}\text { WOR1 (0.806), WOR2 (0.881), WOR3 (0.875), WOR4 (0.871), WOR5 } \\
(0.861) .\end{array}$ & $\mathrm{WOR}=0.859$ \\
\hline WMA & $\begin{array}{l}\text { Work } \\
\text { management }\end{array}$ & $\begin{array}{l}\text { WMA1 (0.843), WMA2 (0.845), WMA3 (0.897), WMA4 (0.885), WMA5 } \\
(0.898) \text {. }\end{array}$ & $\mathrm{WMA}=0.874$ \\
\hline WEN & $\begin{array}{l}\text { Working } \\
\text { environment }\end{array}$ & $\begin{array}{l}\text { WEN1 (0.829), WEN2 (0.860), WEN3 (0.898), WEN4 (0.859), WEN5 } \\
(0.845) \text {. }\end{array}$ & $\mathrm{WEN}=0.858$ \\
\hline WCN & $\begin{array}{l}\text { Working } \\
\text { conditions }\end{array}$ & $\begin{array}{l}\text { WCN1 (0.781), WCN2 (0.774), WCN3 (0.755), WCN4 (0.856), WCN5 } \\
(0.820) \text {. }\end{array}$ & $\mathrm{WCN}=0.797$ \\
\hline
\end{tabular}

Notes: a. 1 components extracted. Extraction Method: Principal Component Analysis.

\section{Results and Discussion}

All the hypotheses were tested performing linear regression, choosing the socially responsible organisation's behaviour and the socially responsible employee's behaviour as dependent variables (Tables 3-4).
In the case of Lithuania, when such features as employee intentions to leave the job, the work content directed to extremes (work tasks exceeding employees' physical abilities and possessed qualifications; tasks degrading the employee's self-esteem; meaningless work tasks; tasks that keep changing too often; i.e., new tasks 
every time, etc.), biased work management, unsatisfactory working environment (e.g., working environment that does not correspond to ergonomic requirements), and poor physical working conditions are weakening and other variables remain unchanged, socially responsible organisational behaviour (FOSB) is strengthening. In turn, in Lithuanian organisations, as the negative nature of tasks is decreasing (i.e., tasks harmful to the employee's health, tasks not conforming to universally accepted moral norms, etc.) and other remaining variables remain unchanged, employees' social responsibility (FESB) is strengthening (Table 3).

Regression equations (Lithuania):

$\mathrm{FOSB}=5.093-0.105 * \mathrm{EIN}-0.079 * \mathrm{WTA}-0.249 *$ WMA - $0.123 *$ WEN $-0.314 *$ WCN.

$\mathrm{FESB}=4.100-0.136 *$ WTA.

In the case of Poland, when such features as employees' negative attitudes and intentions are weakening, while work organisation and management, the working environment and working conditions are improving, socially responsible organisational behaviour (FOSB) is also strengthening. Other variables have no significant effect on this. In the case of Poland, as employees' negative intentions and attitudes are weakening and the nature of tasks and working conditions become more adequate while other remaining variables are unchanging, the employee's socially responsible behaviour (FESB) is also strengthening (Table 4).

Regression equations (Poland):

$\mathrm{FOSB}=5.536-0.076 * \mathrm{EVS}-0.185 * \mathrm{EIN}-0.096 *$ WOR $-0.090 * \mathrm{WMA}-0.147 * \mathrm{WEN}-0.205 * \mathrm{WCN}$.

$\mathrm{FESB}=5.326-0.258 * \mathrm{EVS}-0.116 * \mathrm{EIN}-0.146 *$ WTA - $0.130 *$ WCN.

Having identified the cases of Lithuanian and Polish organisations separately, it further makes sense to compare the two countries with respect to the analysed issue (Table 5). The table shows the relationships between the scale of Corporate Social Responsibility and employees' behaviour subscales.

When negative intentions of employees, inappropriate work management, unsatisfactory working environment and unsatisfactory conditions are one by one individually weakening and other remaining variables are unchanging, CSR in Lithuanian organisations is improving. Furthermore, when employees' negative intentions and negative attitudes are reducing, unsatisfactory working environment and inappropriate working conditions are one by one individually weakening while other remaining variables remain unchanged, CSR in Polish organisations is strengthening. Thus, the removal of three identified disturbances (I - employees' intentions; II - working environment; III - working conditions), between which statistically reliable differences were found in both countries, would contribute to a more effective implementation of Corporate Social Responsibility principles. However, seeking that the implementation of Corporate Social Responsibility in Lithuanian organisations meets the needs of employees, it is worth paying attention to work management, i.e., considering the strategy for changing work management. Meanwhile, in Poland, seeking that CSR implementation meets employees' needs, it is also worth focusing on employees' views (i.e., religious, political, and humanistic views).

Regression equations (Lithuania-LT and Poland-PL):

CSR $(\mathrm{LT})=4.596-0.090 *$ EIN $-0.105 *$ WMA -0.113

* WEN -0.117 * WCN.

CSR $(P L)=5.431-0.167 *$ EVS $-0.150 *$ EIN -0.122

* WEN -0.167 * WCN.

Table 3

Relationships between Corporate Social Responsibility Subscales and Employee behaviour Subscales in Lithuanian Organizations $(\mathrm{N}=\mathbf{4 1 0})$

\begin{tabular}{|c|c|c|c|c|c|c|c|c|c|c|}
\hline & & & \multicolumn{8}{|c|}{ Corporate Social Responsibility } \\
\hline & & & \multicolumn{4}{|c|}{$\begin{array}{c}\begin{array}{c}\text { Socially responsible organisation's } \\
\text { behaviour (FOSB) }\end{array} \\
\end{array}$} & \multicolumn{4}{|c|}{$\begin{array}{c}\text { Socially responsible employee behaviour } \\
\text { (FESB) }\end{array}$} \\
\hline & & & $\approx$ & $\tilde{z}$ & 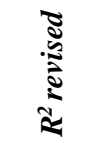 & 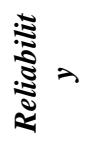 & $\approx$ & $\approx$ & 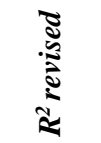 & 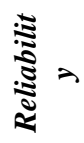 \\
\hline & & & 0.779 & 0.605 & 0.591 & 0.000 & 0.314 & 0.098 & 0.064 & 0.000 \\
\hline \multicolumn{3}{|c|}{ Independent variable } & 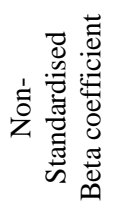 & 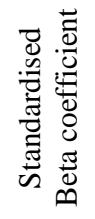 & - & 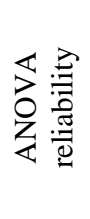 & 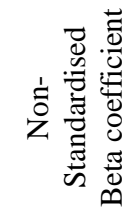 & 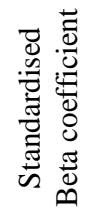 & - & 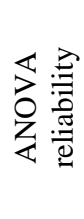 \\
\hline \multicolumn{3}{|c|}{ (Constant) } & 5.093 & & 31.550 & 0.000 & 4.100 & & 18.868 & 0.000 \\
\hline \multirow{5}{*}{ FEIR } & $\begin{array}{l}\text { Employee } \\
\text { communication }\end{array}$ & ECO & -0.036 & -0.030 & -0.611 & 0.541 & 0.120 & 0.111 & 1.505 & 0.133 \\
\hline & $\begin{array}{l}\text { Employee } \\
\text { isolation }\end{array}$ & EIS & 0.050 & 0.048 & 0.768 & 0.443 & 0.004 & 0.005 & 0.050 & 0.960 \\
\hline & $\begin{array}{l}\text { Employee } \\
\text { reputation }\end{array}$ & ERE & -0.052 & -0.054 & -0.927 & 0.354 & -0.017 & -0.021 & -0.231 & 0.818 \\
\hline & $\begin{array}{l}\text { Employee } \\
\text { demography }\end{array}$ & EDE & -0.014 & -0.010 & -0.193 & 0.847 & -0.146 & -0.115 & -1.512 & 0.131 \\
\hline & Employee views & EVS & -0.053 & -0.043 & -0.926 & 0.355 & 0.039 & 0.036 & 0.512 & 0.609 \\
\hline
\end{tabular}


Jolita Vveinhardt, Wlodzimierz Sroka. Independent Variables Affecting Employee Behaviour in Socially Responsible...

\begin{tabular}{|c|c|c|c|c|c|c|c|c|c|c|}
\hline & & & \multicolumn{8}{|c|}{ Corporate Social Responsibility } \\
\hline & & & \multicolumn{4}{|c|}{$\begin{array}{c}\text { Socially responsible organisation's } \\
\text { behaviour (FOSB) }\end{array}$} & \multicolumn{4}{|c|}{$\begin{array}{c}\text { Socially responsible employee behaviour } \\
\text { (FESB) }\end{array}$} \\
\hline & & & $\approx$ & $\approx$ & 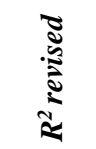 & 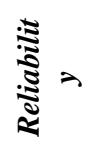 & $\approx$ & $\tilde{z}$ & 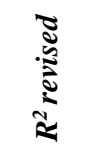 & 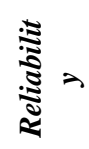 \\
\hline & & & 0.779 & 0.605 & 0.591 & $\mathbf{0 . 0 0 0}$ & 0.314 & 0.098 & 0.064 & $\mathbf{0 . 0 0 0}$ \\
\hline \multicolumn{3}{|c|}{ Independent variable } & 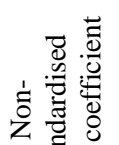 & 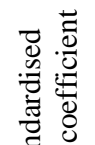 & - & 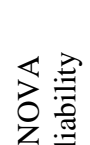 & 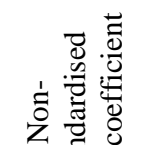 & 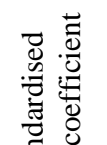 & - & 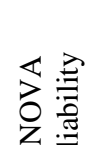 \\
\hline & $\begin{array}{l}\text { Damage } \\
\text { experienced by } \\
\text { employees }\end{array}$ & EED & 0.048 & 0.045 & 0.898 & 0.370 & -0.087 & -0.092 & -1.221 & 0.223 \\
\hline & $\begin{array}{l}\text { Employees' } \\
\text { emotional state }\end{array}$ & EES & 0.007 & 0.009 & 0.134 & 0.893 & 0.052 & 0.067 & 0.692 & 0.489 \\
\hline & $\begin{array}{l}\text { Employee } \\
\text { intentions }\end{array}$ & EIN & -0.105 & -0.148 & -2.720 & 0.007 & -0.075 & -0.119 & -1.448 & 0.148 \\
\hline \multirow{3}{*}{ FNCA } & Nature of tasks & WTA & 0.085 & 0.079 & 1.698 & 0.090 & -0.136 & -0.143 & -2.031 & 0.043 \\
\hline & Work content & WCT & -0.079 & -0.070 & -1.938 & 0.050 & 0.008 & 0.008 & 0.144 & 0.885 \\
\hline & $\begin{array}{l}\text { Work } \\
\text { assessment }\end{array}$ & WAS & 0.009 & 0.011 & 0.216 & 0.829 & -0.091 & -0.124 & -1.567 & 0.118 \\
\hline \multirow{2}{*}{ FWOM } & $\begin{array}{l}\text { Work } \\
\text { organisation }\end{array}$ & WOR & -0.027 & -0.030 & -0.563 & 0.574 & 0.104 & 0.131 & 1.596 & 0.111 \\
\hline & $\begin{array}{l}\text { Work } \\
\text { management }\end{array}$ & WMA & -0.249 & -0.287 & -5.258 & 0.000 & 0.038 & 0.050 & 0.601 & 0.548 \\
\hline \multirow{2}{*}{ FPEC } & $\begin{array}{l}\text { Working } \\
\text { environment }\end{array}$ & WEN & -0.123 & -0.135 & -2.778 & 0.006 & -0.103 & -0.127 & -1.728 & 0.085 \\
\hline & $\begin{array}{l}\text { Working } \\
\text { conditions }\end{array}$ & WCN & -0.314 & -0.359 & -7.580 & 0.000 & 0.079 & 0.102 & 1.425 & 0.155 \\
\hline
\end{tabular}

Note: $\mathrm{R}$ - set correlation coefficient; $\mathrm{R}^{2}$ - aggregate coefficient of certainty (coefficient of determination); $\mathrm{F}$ - observed value of Fisher's statistics.

Table 4

Relationships between Corporate Social Responsibility Subscales and Employee behaviour Subscales in Polish Organisations $(\mathrm{N}=\mathbf{4 1 3})$

\begin{tabular}{|c|c|c|c|c|c|c|c|c|c|c|}
\hline & & & \multicolumn{8}{|c|}{ Corporate Social Responsibility } \\
\hline & & & \multicolumn{4}{|c|}{$\begin{array}{c}\text { Socially responsible organisation's } \\
\text { behaviour (FOSB) }\end{array}$} & \multicolumn{4}{|c|}{$\begin{array}{c}\text { Socially responsible employee } \\
\text { behaviour (FESB) }\end{array}$} \\
\hline & & & $\approx$ & है & 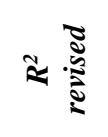 & 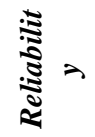 & $\approx$ & है & 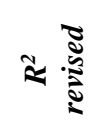 & 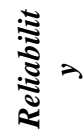 \\
\hline & & & 0.839 & 0.703 & 0.692 & 0.000 & 0.605 & 0.366 & 0.342 & 0.000 \\
\hline \multicolumn{3}{|c|}{ Independent variable } & 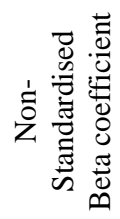 & 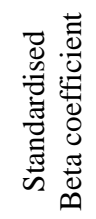 & - & 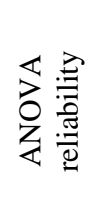 & 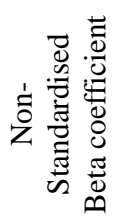 & 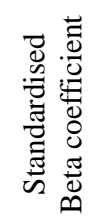 & - & 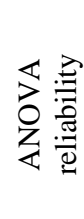 \\
\hline \multicolumn{3}{|c|}{ (Constant) } & 5.536 & & 53.936 & 0.000 & 5.326 & & 35.889 & 0.000 \\
\hline \multirow{6}{*}{ FEIR } & $\begin{array}{l}\text { Employee } \\
\text { communication }\end{array}$ & ECO & 0.038 & 0.041 & 0.750 & 0.453 & 0.002 & 0.002 & 0.023 & 0.982 \\
\hline & Employee isolation & EIS & -0.015 & -0.018 & -0.273 & 0.785 & 0.092 & 0.107 & 1.138 & 0.256 \\
\hline & Employee reputation & ERE & -0.056 & -0.066 & -1.187 & 0.236 & 0.008 & 0.009 & 0.114 & 0.910 \\
\hline & $\begin{array}{l}\text { Employee } \\
\text { demography }\end{array}$ & EDE & -0.001 & -0.001 & -0.015 & 0.988 & 0.000 & 0.000 & 0.006 & 0.995 \\
\hline & Employee views & EVS & -0.076 & -0.084 & -1.975 & 0.049 & -0.258 & -0.290 & -4.648 & 0.000 \\
\hline & $\begin{array}{l}\text { Damage experienced } \\
\text { by employees }\end{array}$ & EED & -0.047 & -0.055 & -1.406 & 0.161 & 0.003 & 0.004 & 0.065 & 0.948 \\
\hline
\end{tabular}




\begin{tabular}{|c|c|c|c|c|c|c|c|c|c|c|}
\hline & & & \multicolumn{8}{|c|}{ Corporate Social Responsibility } \\
\hline & & & \multicolumn{4}{|c|}{$\begin{array}{c}\text { Socially responsible organisation's } \\
\text { behaviour (FOSB) }\end{array}$} & \multicolumn{4}{|c|}{\begin{tabular}{|c|} 
Socially responsible employee \\
behaviour (FESB)
\end{tabular}} \\
\hline & & & $\approx$ & $\approx$ & $\approx \frac{\bar{\Sigma}}{2}$ & 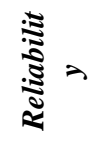 & $\approx$ & $\approx$ & $\approx \frac{\tilde{\Sigma}}{\frac{2}{2}}$ & 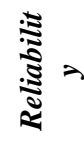 \\
\hline & & & 0.839 & 0.703 & 0.692 & 0.000 & 0.605 & 0.366 & 0.342 & 0.000 \\
\hline \multicolumn{3}{|c|}{ Independent variable } & 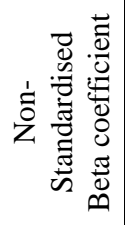 & 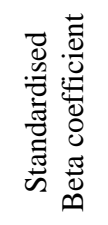 & - & 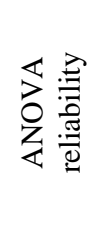 & 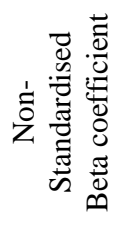 & 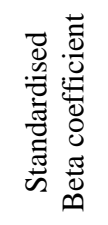 & - & 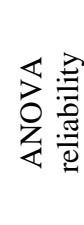 \\
\hline & $\begin{array}{l}\text { Employees' } \\
\text { emotional state }\end{array}$ & EES & 0.047 & 0.059 & 1.206 & 0.228 & 0.081 & 0.104 & 1.440 & 0.151 \\
\hline & Employee intentions & EIN & -0.185 & -0.275 & -5.571 & 0.000 & -0.116 & -0.174 & -2.413 & 0.016 \\
\hline \multirow{3}{*}{ FNCA } & Nature of tasks & WTA & 0.022 & 0.029 & 0.563 & 0.574 & -0.146 & -0.197 & -2.637 & 0.009 \\
\hline & Work content & WCT & -0.031 & -0.035 & -0.938 & 0.349 & -0.082 & -0.094 & -1.711 & 0.088 \\
\hline & Work assessment & WAS & -0.025 & -0.039 & -0.790 & 0.430 & -0.050 & -0.076 & -1.066 & 0.287 \\
\hline \multirow{2}{*}{ FWOM } & Work organisation & WOR & -0.096 & -0.122 & -2.721 & 0.007 & -0.015 & -0.019 & -0.296 & 0.767 \\
\hline & Work management & WMA & -0.090 & -0.105 & -2.195 & 0.029 & 0.038 & 0.046 & 0.650 & 0.516 \\
\hline \multirow[t]{2}{*}{ FPEC } & $\begin{array}{l}\text { Working } \\
\text { environment }\end{array}$ & WEN & -0.147 & -0.181 & -3.899 & 0.000 & -0.098 & -0.123 & -1.810 & 0.071 \\
\hline & Working conditions & WCN & -0.205 & -0.246 & -5.441 & 0.000 & -0.130 & -0.158 & -2.379 & 0.018 \\
\hline
\end{tabular}

Note: $\mathrm{R}$ - set correlation coefficient; $\mathrm{R}^{2}$ - aggregate coefficient of certainty (coefficient of determination); F - observed value of Fisher's statistics.

Table 5

Relationships between Corporate Social Responsibility Scale and Employee behaviour Subscales in Lithuanian and Polish Organizations $(\mathbf{N}=823)$

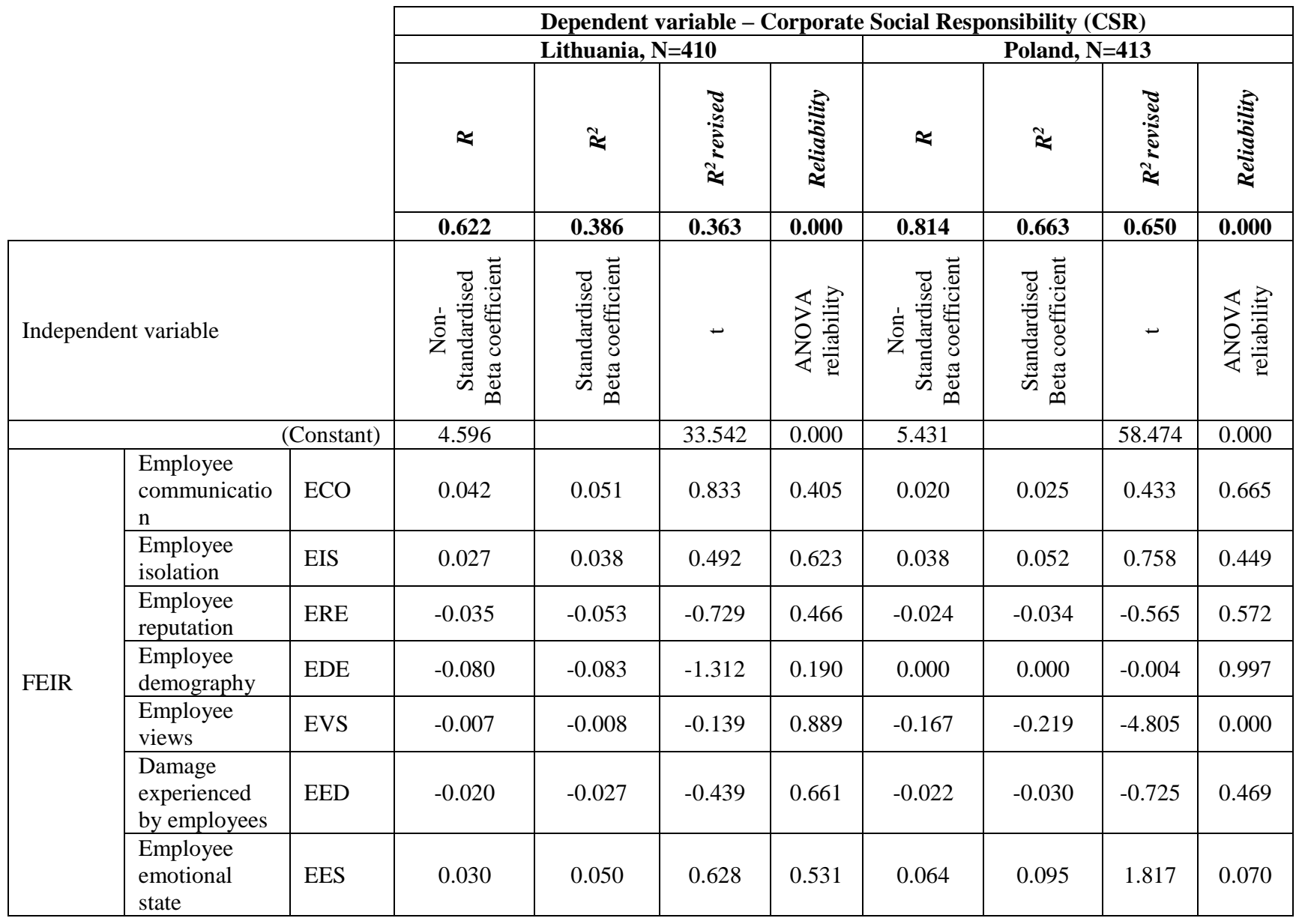




\begin{tabular}{|c|c|c|c|c|c|c|c|c|c|c|}
\hline \multicolumn{11}{|c|}{ Dependent variable - Corporate Social Responsibility (CSR) } \\
\hline & & & \multicolumn{4}{|c|}{ Lithuania, $N=410$} & \multicolumn{4}{|c|}{ Poland, $\mathrm{N}=413$} \\
\hline & & & $\approx$ & $\leftleftarrows$ & 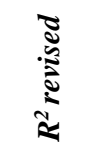 & 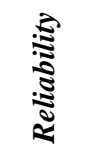 & $\approx$ & 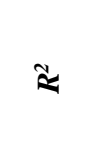 & 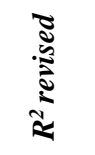 & 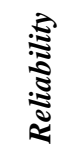 \\
\hline & & & 0.622 & 0.386 & 0.363 & $\mathbf{0 . 0 0 0}$ & 0.814 & 0.663 & 0.650 & 0.000 \\
\hline \multicolumn{3}{|c|}{ Independent variable } & 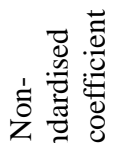 & 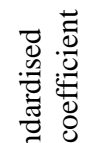 & - & 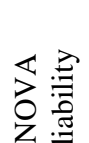 & 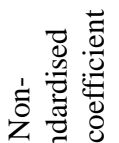 & 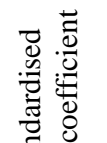 & - & 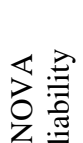 \\
\hline & $\begin{array}{l}\text { Employee } \\
\text { intentions }\end{array}$ & EIN & -0.090 & -0.187 & -2.750 & 0.006 & -0.150 & -0.263 & -5.006 & 0.000 \\
\hline \multirow{3}{*}{ FNCA } & $\begin{array}{ll}\begin{array}{l}\text { Nature } \\
\text { tasks }\end{array} & \text { of } \\
\end{array}$ & WTA & -0.026 & -0.035 & -0.610 & 0.542 & -0.062 & -0.098 & -1.795 & 0.073 \\
\hline & Work content & WCT & 0.044 & 0.056 & 1.256 & 0.210 & -0.057 & -0.076 & -1.885 & 0.060 \\
\hline & $\begin{array}{l}\text { Work } \\
\text { assessment }\end{array}$ & WAS & -0.041 & -0.073 & -1.115 & 0.266 & -0.038 & -0.067 & -1.289 & 0.198 \\
\hline \multirow{2}{*}{ FWOM } & $\begin{array}{l}\text { Work } \\
\text { organisation }\end{array}$ & WOR & 0.038 & 0.063 & 0.934 & 0.351 & -0.055 & -0.083 & -1.740 & 0.083 \\
\hline & $\begin{array}{l}\text { Work } \\
\text { management }\end{array}$ & WMA & -0.105 & -0.178 & -2.621 & 0.009 & -0.026 & -0.035 & -0.694 & 0.488 \\
\hline \multirow{2}{*}{ FPEC } & $\begin{array}{l}\text { Working } \\
\text { environment }\end{array}$ & WEN & -0.113 & -0.182 & -3.007 & 0.003 & -0.122 & -0.179 & -3.601 & 0.000 \\
\hline & $\begin{array}{l}\text { Working } \\
\text { conditions }\end{array}$ & WCN & -0.117 & -0.197 & -3.335 & 0.001 & -0.167 & -0.237 & -4.907 & 0.000 \\
\hline
\end{tabular}

Note: $\mathrm{R}$ - set correlation coefficient; $\mathrm{R}^{2}$ - aggregate coefficient of certainty (coefficient of determination); F - observed value of Fisher's statistics.

The analysis conducted allows to state that:

H1. Along with the improvement of factors related to employee relationships, the socially responsible organisation's behaviour is strengthening (improving). Hypothesis $\mathrm{H1}$ was rejected.

$\mathrm{H} 2$. Along with the improvement of factors related to work management, the socially responsible organisation's behaviour is strengthening (improving). Hypothesis H2 was confirmed in both countries.

These findings are in line with the research results of Kundu and Gahlawat (2016). Analysing 563 respondents from 204 organisations in India, they have revealed that "investments in socially responsible HR practices on the part of organisation are positively perceived by the employees, which, in turn, encourage them to the same through display of positive work-related attitudes".

H3. Along with the improvement of factors related to the working environment and working conditions, the socially responsible organisation's behaviour is strengthening (improving). Hypothesis $\mathrm{H3}$ was confirmed in both countries.

It can be then stated that our results in terms of $\mathrm{H} 2$ and H3 support the findings of Searcy et al. (2016). They stated that greater attention by companies to working conditions could benefit employees, as the socially responsible organization as a rule is more beneficial for its employees. Our study is in line with findings of Coller et al. (2015) who stated that CSR was perceived as an opportunity to improve working conditions (p.151), which improved the economic performance of companies and their competitiveness ( $p$. 162). Our findings also confirm the results of Kabir et al. (2018) who stated that negative workplace was associated with an abnormal decrease in shareholder value. They also added that it had a more pronounced negative effect on firm value in the present environment than previously. In turn, in relation to $\mathrm{H} 1$, our findings are in opposition to the results of Searcy et al. (2016).

H4. Along with the improvement of factors related to the nature of tasks, the socially responsible employee's behaviour is strengthening (improving). Hypothesis H4 was confirmed in both countries.

Interestingly, the results of our survey were very similar in both analysed countries, especially in the light of analyses conducted by other scholars. Kliestikova and Janoskova (2017) have analysed consumer profiles depending on countries. Their surveys have revealed that Slovakia and Czech Republic were grouped in different clusters. It might be surprising because both countries belonged to one country; i.e., to Czechoslovakia for a dozen of years (in the $20^{\text {th }}$ century). Whereas Poland and Lithuania, though being linked through historical and cultural heritage, were in the same situation hundreds of years ago. It must be added, however, that our research focused on other issues.

\section{Conclusions}

This study improves the understanding of socially responsible behaviour of organisations and shows how changes in internal processes and working conditions of organisations can increase employee social responsibility and CSR of the organisations themselves. The results of the study allow us to draw several conclusions, which are closely interrelated. First, fulfilment of commitments to 
employees as internal stakeholders should not be treated only as a certain indicator of social responsibility. Employees respond sensitively to leadership content and working conditions that are an important factor determining the quality of their own behaviour; therefore, positive changes in the internal environment can be a strong stimulus that also increases outward-directed corporate social responsibility. At the same time, this complements the understanding of the stakeholder theory by showing the existing links between the organization's internal policies towards employees and the socially responsible behaviour of employees themselves. In practice, this means that organizations that have improved working conditions and work organisation can expect that the behaviour of employees in the eyes of customers will further confirm good intentions of the enterprise declaring CSR. In our opinion, this can be a significant argument for enterprises as to why they should pay more attention to internal stakeholders. Employees in this case can be treated as certain agents who indirectly inform external stakeholders about the enterprise's responsibility. Second, implementation of internal changes would reduce the gap observed in the scientific literature between declared CSR and actual practice. That is, in practice, CSR is often understood as a certain marketing strategy; therefore, implementation of change would contribute to greater confidence in CSR. Third, to implement the practice of internal change of CSR, one should analyse the attitudes of employees working in organisations of a specific country, which can reduce costs of implementing corporate social responsibility. Our study has demonstrated that it is beneficial to first evaluate how employees respond to existing leadership, work tasks, and working conditions.

Organisations should in principle consider the strategy of work management, specifically of work content, namely paying attention to the adequacy of tasks given to employees and the time allocated for their performance, avoiding duplication of work and changing of tasks in the course of carrying them out. The physical working environment and assurance of ergonomic working conditions are obligatory for all organisations regardless of their CSR status. However, the results of the study demonstrate that organisations show insufficient concern for employees' physical well-being and safety.

It is likely that the results of the study could affect corporate social responsibility towards improvement when more attention is paid to the employee, because the organisation's social behaviour is directly related to the socially responsible employee.

In addition to what has been mentioned above, our paper contributes to science in the following way. First, its international aspect, which is much more interesting (and valuable) than a one-country analysis, must be emphasised.
This can be seen as an important advantage of the study as it provides new knowledge needed for a better understanding of CSR in Central and Eastern Europe. To our knowledge, CSR practice in such aspects has not been investigated in this region. Moreover, our study analyses a sample consisting of 823 respondents employed in both private and public organisations, ensuring a sufficient number of respondents in each country, which allows extrapolating the results to both populations. Second, it improves our understanding of variables affecting employees' behaviour in socially responsible organisations. Considering that, an opportunity to improve CSR policies of individual organizations is created.

When undertaking the study, we did not set ourselves the objective of discovering differences between the two countries; i.e., our aim was to identify independent variables in the case of each of two geographically, historically, and culturally close countries. Therefore, we propose that in the future, research studies of two or more countries are compared, especially the ones that are not geographically, historically and culturally close to each other.

Of course, our paper is not without limitations. The first is that it is of a quantitative nature, and we believe that a qualitative survey could also bring interesting feedback on the topic being analysed. The second limitation refers to the countries analysed. As stated earlier, two historically and culturally close countries were chosen for the analysis. Hence, it could have been assumed - at least to some extent - that the findings would be similar. This assumption was soundly confirmed by the findings obtained. Given this fact, it could have been assumed that the results would be different when analysing culturally different countries; e.g., those of different continents. Third, the perception of CSR and employee's behaviour is constantly evolving in time. For example, Hamzaoglu et al. (2021) pointed out that societal culture and employee knowledge of what behaviours can be treated as mobbing influence individuals' levels of mobbing perception. This was not assessed in this study, and the increasing employee awareness of ethical behaviour may change the findings. That is why cyclical research conducted every 5-10 years would assist in observations of the direction in which the said phenomenon evolves. Furthermore, our study shows only what influences better employee attitude towards CSR, but in the future it would make sense to examine in more detail how this affects customer satisfaction and their opinion about the organization's social responsibility. It would also be meaningful to assess the additional influence of social and demographic factors. It must be noted that this study provides only an overall picture that would be better detailed by gender and age factors, and it would also be beneficial to investigate private and public sectors separately.

\section{Acknowledgements}

The publication of the article was funded under the auspices of the programme of the Minister of Science and Higher Education in Poland entitled "Regional Initiative of Excellence" in 2019-2022, project number 018/RID/2018/19; amount of funding: PLN 10,788,423.16. 


\section{References}

Ahmad, I., Donia, M. B. L., Shahzad, K. (2019). Impact of Corporate Social Responsibility Attributions on Employees' Creative Performance: The Mediating Role of Psychological Safety. Ethics \& Behavior, 29(6), 490-509. https://doi.org/10.10 80/10508422.2018.1501566

Akar, H. (2018). Meta-analysis study on organizational outcomes of ethical leadership. Educational Policy Analysis and Strategic Research, 13(4), 6-25. https://doi.org/10.29329/epasr.2018.178.1

Aluchna, M., \& Roszkowska-Menkes, M. (2019). Integrating Corporate Social Responsibility and Corporate Governance at the Company Level, Towards a Conceptual Model. Inzinerine Ekonomika-Engineering Economics, 30(3), 349-361. https://doi.org/10.5755/j01.ee.30.1.14306

Androniceanu, A. (2019). Social responsibility, an essential strategic option for a sustainable development in the field of bioeconomy. Amfiteatru Economic, 21(52), 503-519. https://doi.org/10.24818/EA/2019/52/503

Androniceanu, A., \& Tvaronaviciene, M. (2019). Developing a holistic system for social assistance services based on effective and sustainable partnerships. Administration \& Public Management Review, 33, 103-118. https://doi.org/10.24 818/amp/2019.33-06.

Anjum, A., Ming, X., Siddiqi, A. F., \& Rasool, S. F. (2018). An Empirical Study Analyzing Job Productivity in Toxic Workplace Environments. International Journal of Environmental Research and Public Health, 15(5), 1035. https://doi.org/10.33 90/ijerph15051035.

Bhattacharya, S. (2017). Does Corporate Social Responsibility Contribute to strengthen brand equity? An empirical study, International Review on Public and Nonprofit Marketing, 14(4), 513-533. https://doi.org/10.1007/s12208-017-0185-z

Chapardar, H., \& Khanlari, R. (2011). Iranian corporations and corporate social responsibility: an overview to adoption of CSR Themes. SAGE Open, 1(3), 1-15. https://doi.org/10.1177\%2F2158244011430988

Cheshire, L. A. (2010). Corporate responsibility? The constitution of fly-in, fly-out mining companies as governance partners in remote, mine-affected localities. Journal of Rural Studies, 26(1), 12-20. https://doi.org/10.1016/j.jrurstud.2009.06.005

Chwistecka-Dudek, H. (2016). Corporate Social Responsibility: supporters vs. opponents of the concept. Forum Scientiae Oeconomia, 4(4), 171-179.

Coller, X., Combra-Fierro, J., Gualtieri, T., \& Melero-Polo, I. (2015). An opportunity to improve working conditions through CSR, In L. Preuss, M. Gold, Ch. Rees (Eds.). Corporate Social Responsibility and Trade Unions: Perspectives across Europe (pp. 151-167), Routledge: London (UK)

Czubala, A. (2016). Corporate Social Responsibility in marketing. Forum Scientiae Oeconomia, 4(1), $103-111$.

De Roeck, K., \& Farooq, O. (2018). Corporate Social Responsibility and Ethical Leadership: Investigating Their Interactive Effect on Employees' Socially Responsible Behaviors. Journal of Business Ethics, 151, 923-939. https://doi.org/10.1007/s10551-017-3656-6

Garavan, T. N., Heraty, N., Rock, A., \& Dalton, E. (2010). Conceptualizing the behavioral barriers to CSR and CS in organizations: A typology of HRD interventions. Advances in Developing Human Resources, 12(5), 587-613. https://doi.org/10.1177/1523422310394779

Gkorezis, P., \& Petridou, E. (2017). Corporate Social Responsibility and pro-environmental behaviour: organisational identification as a Mediator. European Journal of International Management, 11(1), 1-18. https://doi.org/10.150 4/EJIM.2017.081248

Goel, M., \& Ramanathan, P. E. (2014). Business ethics and Corporate Social Responsibility - Is there a dividing line? Procedia Economics and Finance, 11, 49-59. https://doi.org/10.1016/S2212-5671(14)00175-0

Hamzaoglu, N., Yayak, A., \& Turk, B. (2021). Evaluation of mobbing perception levels of health employees. Health Services Management Research. https://doi.org/10.1177/09514848211001689

Hansen, S. D., Dunford, B. B., Alge, B. J., \& Jackson, C. L. (2016). Corporate Social Responsibility, Ethical Leadership, and Trust Propensity: A Multi-Experience Model of Perceived Ethical Climate. Journal of Business Ethics, 137, 649-662. https://doi.org/10.1007/s10551-015-2745-7

Kabir, Q. S., Watson, K., \& Somaratna, T. (2018). Workplace safety events and firm performance. Journal of Manufacturing Technology Management, 29(1), 104-120. https://doi.org/10.1108/JMTM-07-2017-0133

Khan, M. A., Gu, L., Khan, M. A., \& Olah, J. (2020). Natural resources and financial development: The role of institutional quality. Journal of Multinational Financial Management, 56, 100641. https://doi.org/10.1016/j.mulfin.2020.100641

Khan, N., Ahmad, I., \& Ilyas, M. (2018). Impact of ethical leadership on organizational safety performance: The mediating role of safety culture and safety consciousness. Ethics \& Behavior, 28(8), 628-643. https://doi.org/10/1080/1050842 2.2018.1427097.

Kliestikova, J., \& Janoskova, K. (2017). Branding with understanding: How national profile of consumer influences brand value perception. Marketing and Management of Innovations, 3, 149-157. https://doi.org/10.21272/mmi.2017.3-14 
Kuenzi, M., \& Schminke, M. (2009). Assembling fragments into a Lens: a review, critique, and proposed research agenda for the organizational work climate literature, Journal of Management, 35(3), 634-717. https://psycnet.apa.org/doi/10.11 77/0149206308330559

Kundu, S. C., \& Gahlawat, N. (2016). Effects of socially responsible HR practices on employees' work attitudes. International Journal of Human Resources Development and Management, 16(3/4), 140-160. https://doi.org/10.1504/IJH RDM.2016.078194

Kyun Chang, Y., Lee, S., \& Won-Yong Oh, W. Oh. (2021). The impact of CEOs' regulatory focus on CSR: the strengthening effects of industry-level dynamism and firm-level volatility. European Journal of International Management, 15(4), 511538. https://doi.org/10.1504/EJIM.2021.114662

Lee, E. M., Park, S., \& Lee, H. (2013). Employee perception of CSR activities: Its antecedents and consequences. Journal of Business Research, 66, 1716-1720. https://doi.org/10.1016/j.jbusres.2012.11.008

Lee, Y. (2020). Toward a communality with employees: The role of CSR types and internal reputation. Corporate Reputation Review, 23, 13-23. https://doi.org/10.1057/s41299-019-00069-x

Leymann, H. (1990). Mobbing and Psychological Terror at Workplaces. Violence and Victims, 5(2), $119-126$. https://doi.org/10.1891/0886-6708.5.2.119

Lutgen-Sandvik, P., \& Tracy, S. J. (2012). Answering Five Key Questions about Workplace Bullying: How Communication Scholarship Provides Thought Leadership for Transforming Abuse at Work. Management Communication Quarterly, 26(1), 3-47. https://doi.org/10.1177/0893318911414400

Macassa, G., McGrath, C., Tomaselli, G., \& Buttigieg, S. C. (2020). Corporate social responsibility and internal stakeholders' health and well-being in Europe: A systematic descriptive review. Health Promotion International, 1-18. https://doi.org/10.1093/heapro/daaa071

Majerova, J. (2015). Analysis of Slovak consumer's perception of the green marketing activities. Procedia Economics and Finance, 26, 553-560. DOI: 10.1016/S2212-5671(15)00954-5

McCarthy, P., Sheehan, M., Barker, M., \& Henderson, M. (2003). Ethical investment and workplace bullying: consonances and dissonances', International Journal of Management and Decision Making, 4(1), 11-23. https://doi.org/10.1504/IJMD M.2003.002485

Mohr, L. A., Webb, D. J., \& Harris, K. E. (2001). Do consumers expect companies to be socially responsible? The impact of Corporate Social Responsibility on buying behaviour, Journal of Consumer Affairs, 1(35), 45-72. https://doi.org/10.11 11/j.1745-6606.2001.tb00102.x

Moratis, L. (2016) Out of the ordinary? Appraising ISO 26000's CSR definition, International Journal of Law and Management, 58(1), 26-47. https://doi.org/10.1108/IJLMA-12-2014-0064

Moratis, L. (2018). Signalling Responsibility? Applying Signalling Theory to the ISO 26000 Standard for Social Responsibility, Sustainability, 10(11), 4172. https://doi.org/10.3390/su10114172

Nahrgang, J. D., Morgeson, F., \& Hofmann, D. (2011). Safety at work: a meta-analytic investigation of the link between job demands, job resources, burnout, engagement, and safety outcomes. The Journal of Applied Psychology, 96(1), 71-94. https://doi.org/10.1037/a0021484

Nejati, M., Salamzadeh, Y., \& Loke, Ch. K. (2019). Can ethical leaders drive employees' CSR engagement? Social Responsibility Journal, 16(5), 655-669. https://doi.org/10.1108/srj-11-2018-0298

Neumann, W. P., Dixon, S. M., \& Nordvall, A. C. (2014). Consumer demand as a driver of improved working conditions: the 'Ergo-Brand' proposition. Ergonomics, 57(8), 1113-1126. https://doi.org/10.1080/00140139.2014.917203

O'Connor, A., \& Shumate, M. (2010). An economic industry and institutional level of analysis of corporate social responsibility communication. Management Communication Quarterly, 24(4), 529-551. https://doi.org/10.1177\%2F0893318 909358747

Ostroff, C., Kinicki, A. J., \& Muhammad, R. S. (2012). Organizational Culture and Climate. Handbook of Psychology, 12, 643676. https://doi.org/10.1002/9781118133880.hop212024.

Petrescu, C., \& Manghiuc, I. (2020). Mobbing at Work and the Impact of Employee Performance', In A. Grigorescu \& V. Radu (Ed.), Lumen Proceedings: 11. 1st International Conference Global Ethics - Key of Sustainability (GEKoS) (pp. 273-282). Iasi, Romania: LUMEN Publishing House, 2020. https://doi.org/10.18662/lumproc/gekos2020/28

Rajak, D. (2008). Uplift and empower: The market, morality and corporate responsibility on South Africa's platinum belt', In Geert De Neve, Luetchford Peter, Jeffrey Pratt, Donald C. Wood (eds.), Hidden Hands in the Market: Ethnographies of Fair Trade, Ethical Consumption, and Corporate Social Responsibility. Research in Economic Anthropology, 28, $297-324$. https://doi.org/10.1016/S0190-1281(08)28013-3

Ranki, C., Vrbka, J., Valaskova, K., \& Olah, J. (2018). Objectifying women's bodies in the workplace: gender-based misconduct, egregious sexual pressure, and misogynistic practices. Contemporary Readings in Law and Social Justice, 10(2), 71-77. https://doi.org/10.22381/CRLSJ10220186 
Rose, J. M. (2007). Corporate Directors and Social Responsibility: Ethics versus Shareholder Value. Journal of Business Ethics, 73, 319-331. https://doi.org/10.1007/s10551-006-9209-z

Salciuviene, R., Hopeniene, R., \& Dovaliene, A. (2016). Perceived Corporate Social Responsibility and its Implementation in Practice: The Case of Lithuanian Small and Medium-Sized Enterprises. Inzinerine Ekonomika-Engineering Economics, 27(4), 479-490. https://doi.org/10.5755/j01.ee.27.4.14713

Searcy, C., Dixon, S. M., \& Neumann, W. P. (2016). The use of work environment performance indicators in corporate social responsibility reporting. Journal of Cleaner Production, 112(4), 2907-2921. https://doi.org/10.1016/j.jclepro.2015.10.081.

Sheehy, B. (2015). Defining CSR: Problems and Solutions. Journal of Business Ethics, 131, 625-648. https://doi.org/10.10 07/s10551-014-2281-x

Sroka, W., \& Lorinczy, M. (2015). The perception of ethics in business: analysis of research results. Procedia Economics and Finance, 34, 156-163. https://doi.org/10.1016/S2212-5671(15)01614-7

Sroka, W., \& Vveinhardt, J. (2020). Is a CSR policy an equally effective vaccine against workplace mobbing and psychosocial stressors? International Journal of Environmental Research and Public Health, 17(19), 7292; https://doi.org/10.33 90/ijerph17197292.

Toleikiene, R., \& Jukneviciene, V. (2019). The formation of an integral system of ethics management in local self-government: the approach of political and administrative levels. Forum Scientiae Oeconomia, 7(2), 89-106. https://doi.org/10.23 762/FSO_VOL7_NO2_6

Verdorfer, A. P., Weber, W. G., Unterrainer, Ch., \& Seyr, S. (2013). The relationship between organizational democracy and socio-moral climate: Exploring effects of the ethical context in organizations. Economic and Industrial Democracy, 34(3), 423-449. https://doi.org/10.1177\%2F0143831X12450054

Vveinhardt, J., \& Sroka, W. (2020a). Workplace Mobbing in Polish and Lithuanian Organisations with Regard to Corporate Social Responsibility, International Journal of Environmental Research and Public Health, $17,2944$. https://doi.org/10.3390/ijerph17082944

Vveinhardt, J., \& Sroka, W. (2020b). Mobbing and corporate social responsibility: does the status of the organisation guarantee employee wellbeing and intentions to stay in the job? Oeconomia Copernicana, 11(4), 743-778. https://doi.org/10.241 36/oc. 2020.030

Wang, Y. D., \& Hsieh, H. H. (2013). Organizational ethical climate, perceived organizational support, and employee silence: a cross-level investigation. Human Relations, 66(6), 783-802. https://doi.org/10.1177\%2F0018726712460706

Wang, Y. M., Ahma, W., Arsha, M., Yin, H. L., Ahmed, B., \& Ali, Z. (2021). Impact of Coordination, Psychological Safety, and Job Security on Employees' Performance: The Moderating Role of Coercive Pressure. Sustainability, $13(6), 3175$. https://doi.org/10.3390/su13063175

\section{Authors' biographies}

Jolita Vveinhardt, $\mathrm{PhD}$, is a full professor in the field of Management Science. She works at Vytautas Magnus University and Lithuanian Sports University (Lithuania), as well as WSB University, Dąbrowa Gornicza (Poland). She is heading three scientific groups: "Neuro-Relationships" (Lithuanian Sports University), "Managerial Solutions to Violence in Sport" (Lithuanian Sports University), "The Group of Interdisciplinary Research on Working Environment" (Vytautas Magnus University). She is the author and co-author of more than 200 scientific articles, 100 of which were published in peer reviewed journals of Web of Science Core Collection database. She is a member of the International Baltic Management Development Association, Lithuanian Educational Research Association, Lithuanian Association for Quality Management and Innovations, Lithuanian Trauma Psychology Association, European Society for Traumatic Stress Studies, Lithuanian Neuroscience Association, Lithuanian Ergonomics Association and Federation of European Neuroscience Societies.

Wlodzimierz Sroka, $\mathrm{PhD}$, is an associate professor of strategic management. He is Deputy Head of the Management Department of WSB University in Dąbrowa Gornicza (Poland), extraordinary professor at North-West University (South Africa) as well as visiting professor at University of Johannesburg (South Africa). He is the author of numerous scientific papers about strategic alliances, network organizations, strategy, restructuring of the company, CSR and business ethics, marketing and customer's behaviour, public management as well as innovations and entrepreneurship. He is Editor in Chief at Forum Scientiae Oeconomia (Scopus) and Deputy Editor in Chief at European Journal of International Management (IF = 2,145). He is also a member of Editorial Boards of numerous scientific journals indexed in Web of Science Core Collection and SCOPUS.

The article has been reviewed. Received in March 2021; accepted in June 2021.

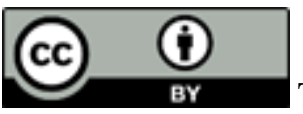

This article is an Open Access article distributed under the terms and conditions of the Creative Commons Attribution 4.0 (CC BY 4.0) License (http://creativecommons.org/licenses/by/4.0/). 